\title{
A METHOD-OF-LINES BASED EFFICIENT METHOD FOR OPTICAL WAVEGUIDE DISCONTINUITY PROBLEMS
}

\author{
Yih-Peng Chiou* and Hung-chun Chang \\ Department of Electrical Engineering and \\ Graduate Institute of Electro-Optcial Engineering \\ National Taiwan University \\ Taipei, Taiwan 106-17, China
}

Abstract-By using the Padé approximation, an efficient method is proposed for the analysis of the optical waveguide discontinuity problem. The eigenvalue problem of the original formulation based on the method of lines is replaced by a rational function of the characteristic matrix. The computation time and memory are further reduced by adopting the multistep method.

\section{Introduction}

Discontinuity problems are important in the investigation of optical devices, such as laser facets, gratings, waveguide ends, and connections of different waveguides. Ikegami [1] solved the laser facet problem by eigenmode expansion and mode matching. Later, many efforts [2]-[9] have been made, which contribute greatly to the understanding of the reflection and transmission phenomena at the discontinuity interface.

The analysis methods reported can be divided into two categories from the viewpoint of linear space bases. Many methods employ the eigenmode expansion method, in which the discrete guided mode(s), the continuous radiation modes, and the continuous evanescent modes are taken as the bases [3]. The reflection and transmission are solved through the mode matching procedure. Using such proper bases, accurate results can be obtained by including relatively smaller number of bases. For example, Ikegami [1] used two bounded bases and explained well the reflection phenomena at a laser facet. However, such methods require mode solving in advance, which is usually a complicated procedure except for such simple structure as the double heterojunction (DH) laser facet. On the other hand, some methods take well-known functions such as sinusoidal functions in the Fourier transform technique and Gaussian functions as bases due to their similarity to the guided modes. Well-known properties of these functions make the analysis easier. Nevertheless, since these bases are not the exact ones for the problem, we need larger number-of bases to achieve accurate results.

In this paper we propose a novel method in which the Padé approximants (PAs) are used. The original formulation is derived from the method of lines (MoL) [9], [10]. Due to the inefficiency of solving eigen systems (that is, mode solving) in the MoL, we approximate the formulation by using the PAs. In the proposed method exact bases are used but mode solving is not necessary Accurate results can be obtained efficiently with low order Padé approximants.

\section{Theory}

Consider TE waves in a nonmagnetic and $z$-invariant medium. The $y$-polarized electric field, $E_{y}$, satisfies the Helmholtz equation

$$
\frac{\partial^{2} E_{y}}{\partial x^{2}}+\frac{\partial^{2} E_{y}}{\partial z^{2}}+k_{0}^{2} n^{2}(x) E_{y}=0
$$

0-7803-4178-3/97/\$10.00 @ 1997 IEEE 
where $n(x)$ is the refractive index distribution. If we discretize the transverse direction into $N$ nodes, (1) becomes

$$
\frac{d^{2} E}{d z^{2}}+[L] E=0
$$

where $[L]$ is the characteristic matrix and $E$ represents the column vector composed of $N$ nodal electric field after discretization. The fields in regions $A$ and $B$, as shown in Fig. 1(a), can be expressed in terms of the fields at the interface $T$ $(z=0)$. If an incident field $E_{A}^{+}$is launched onto the interface $T$, then by fulfilling the continuity boundary conditions for $E_{y}$ and $d E_{y} / d z$, we have the reflected field $E_{A}^{-}$and the transmitted field $E_{B}^{+}$expressed in terms of the incident field as

$$
E_{A}^{-}=\frac{\sqrt{\left[L_{A}\right]}-\sqrt{\left[\bar{L}_{A}\right]}}{\sqrt{\left[L_{A}\right]}+\sqrt{\left[L_{B}\right]}} E_{A}^{+} \quad \text { and } \quad E_{B}^{+}=\frac{2 \sqrt{\left[L_{A}\right]}}{\sqrt{\left[L_{A}\right]}+\sqrt{\left[L_{B}\right]}} E_{A}^{+} .
$$

Note that $\sqrt{[L]}=[V] \sqrt{[D]}[V]^{-1}$ if $[L]=[V][D][V]^{-1}$, where $[D]$ is a diagonal matrix with its diagonal elements being the eigenvalues of $[L]$ and columns of $[V]$ being the corresponding eigenvectors. These eigenvalues and eigenvectors of $[L]$ are the propagation constants and modes of the structure, respectively. The TM case can be derived similarly. Unfortunately, it is inefficient to solve an eigen system especially for non-Hermitian characteristic matrices.

To avoid solving the eigenvalue problem, we propose PAs to approximate the characteristic matrix $[L]\left(\left[L_{A}\right]\right.$ or $\left.\left[L_{B}\right]\right)$ in $(3)$. That is, $\sqrt{[L]}$ is approximated by a rational function of $[L]$. The Pade approximation has been widely used in the wide-angle beam propagation method (BPM) which was originally derived from the paraxial approximation [11]-[13]. $\sqrt{[L]}$ is approximated by the $m$-th order $\mathrm{PA}$ as

$$
\sqrt{[L]} \approx\left(k_{0} \bar{n}+j F^{m}\right) \equiv P^{m}
$$

where $\bar{n}$ is the reference refractive index in the BPM,

$$
F^{1} \equiv \frac{[L]-k_{0}^{2} \bar{n}^{2}}{2 j k_{0} \bar{n}} \quad \text { and } \quad F^{s+1}=\frac{[L]-k_{0}^{2} \bar{n}^{2}}{2 j k_{0} \bar{n}-F^{s}}
$$

where $s$ is the order of the approximant.

The PA is usually expressed as $P(n, d)$, where $n$ and $d$ are the orders of the numerator and the denominator in the rational function, respectively, and $m=n+d$. Making use of the multistep method [13], we can take advantage of the sparseness of $[L]$, and the computation time and memory are further reduced. Compared with the MoL, the computation time and memory are reduced to $O(N)$ from $O\left(N^{3}\right)$ and $O\left(N^{2}\right)$, respectively. The high efficiency of the proposed method is attributed to the fact that the solving procedure of eigen systems is replaced by matrix multiplications and inversions. Besides, this method is versatile in application because $[L]$ is not restricted to Hermitian matrices.

\section{Numerical Results}

We consider Ikegami's sturcture [1], as shown in Fig. 1(b). The wavelength $\lambda$ is $0.86 \mu \mathrm{m}$, the core width $D$ ranges from 0.05 to $1.00 \mu \mathrm{m}$, and the indices of the core and the cladding are $n_{\text {core }}=3.60$ and $n_{\text {clad }}=3.24$, respectively. The computation window is taken large enough such that the fields are negligible at the boundaries. 
Fig. 2 shows the calculated reflectivity of the fundamental TE wave by the MoL and PAs of different orders. The incident ficld is the fundamental mode in the waveguide. The reflectivity $R_{0}$ is defined as the power ratio of the reflected and incident fundamental modes. It is seen that the PA results converge rapidly to the MoL result (without PA) which is shown as the circles in Fig. 2. Our calculation also shows that when the index difference is small, excellent results can be obtained even by paraxial approximation. Fig. 3 demonstrates the good agreement between the paraxial approximation and the MoL.

Fig. 4 shows the reflectivity of the fundamental mode for TE and TM waves for different index contrasts. The core index is 3.60 and the cladding index is $3.60(1-\Delta)$, with $\Delta$ taken to be $3 \%$ and $10 \%$. The solid and dashed lines are calculated using $P(3,3)$. The circles and stars are the results given in $[6]$ based on multimode analysis. The two results coincide except for the TM wave at $\Delta=10 \%$, which is attributed to the larger $\Delta$ and the discontinuity of $E_{x}$. Better result can be achieved by higher-order PAs. Generally speaking, satisfactory results could be obtained using $P(3,3)$.

\section{Conclusion}

In conclusion, we have proposed a novel method based on the Pade approximation for solving the step discontinuity problems for optical waveguides. The method is efficient, accurate, and versatile in application. The results agree well with those obtained using the multimode analysis and the MoL.

\section{Acknowledgment}

This work was supported by the National Science Council of the Republic of China under grant NSC86-2215-E-002-008.

\section{References}

[1] T. Ikegami, "Reflectivity of mode at facet and oscillation mode in doubleheterostructure injection lasers," IEEE J. Quantum Electron., vol. 8, pp. $470-476,1972$.

[2] L. Lewin, "A method for the calculation of the radiation-pattern and modeconversion properties of a solid-state heterojuntion laser," IEEE Trans. Microwave Theory Tech., vol. MTT-23, pp.576-585, 1975.

[3] M. A. A. Pudensi and L. G. Ferreira, "Method to calculate the reflection and transmission of guided waves," J. Opt. Soc. Am., vol. 72, pp. 126-130, 1982.

[4] J. E. Kardontchik, "Mode reflectivity of narrow stripe-geometry double heterostructure lasers," IEEE J. Quantum Electron., vol. 18, pp. 1279-1286, 1982.

[5] A. Hardy, "Formulation of two-dimensional reflectivity calculations based on the effective-index method," J. Opt. Soc. Am. A, vol. 1, pp. 550-555, 1984.

[6] Q. Liu and W. C. Chew, "Analysis of discontinuities in planar dielectric waveguides: an eigenmode propagation method," IEEE Trans. Microwave Theory Tech., vol. 39, pp. 422-429, 1991.

[7] T. Rozzi, L. Zeppelli, and M. N. Husain, "Radiation modes and step discontinuities in dielectric rib waveguides," IEEE Trans. Microwave Theory Tech., vol. 40 , pp. $1879-1888,1992$.

[8] C. J. Smartt, T. M. Benson, and P. C. Kendall, "Exact analysis of waveguide discontinuities: junctions and laser facets," Electron. Lett., vol. 29, 1352$1353,1993$.

[9] R. Pregla and E. Ahler, "Method of lines for analysis of discontinuities in optical waveguide," Electron. Lett., vol. 29, 1845-1847, 1993. 
[10] R. Pregla and W. Pascher, "The method of lines," in Numerical Techniques for Microwave and Millimeter-Wave Passive Structures, T. Itoh, Ed., New York: Wiley, 1989.

[11] N. A. Fleck, J. R. Morris, and M. D. Feit, "Time-dependent propagation of high energy laser beams through the atmosphere," Appl. Phys., vol. 10, pp. 129-160, 1976.

[12] G. R. Hadley, "Wide-angle beam propagation using Padé approximant operators," Opt. Lett., vol. 17, pp. 1426-1428, 1992.

[13] G. R. Hadley, "Multistep method for wide-angle beam propagation," Opt. Lett., vol. 17, pp. 1743-1745, 1992.

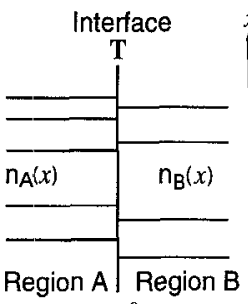

$z=0$

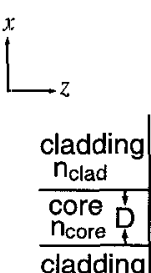

cladding

$\mathrm{n}_{\text {clad }}$

(b)

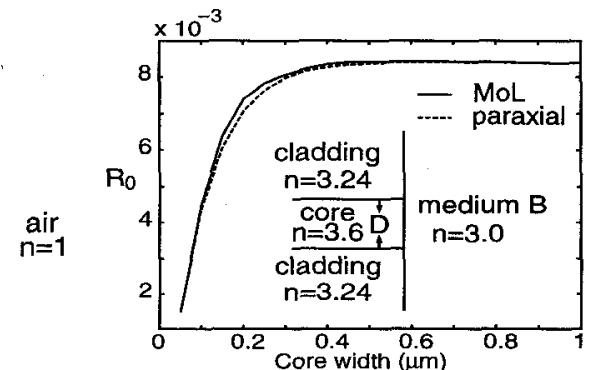

Fig. 1 (a) Layered media A and B seperated by the interface $T$ in the transverse direction. (b) Structure of the laser facet problem.

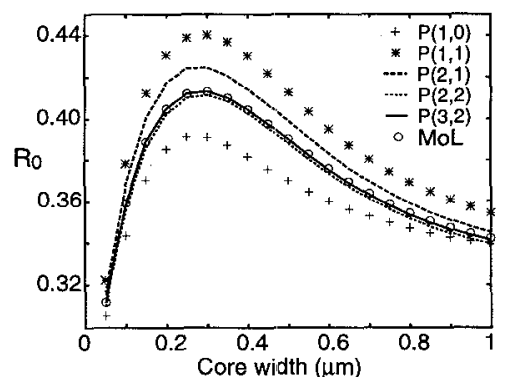

Fig. 2 Reflectivity of the fundamental mode TE wave as a function of the core width, calculated by the MoL and Padé approximants of different orders.

Fig. 3 Reflectivity of the fundamental mode TE wave as a function of the core width, calculated by the paraxial approximation and the MoL. The index difference at the interface is much smaller.

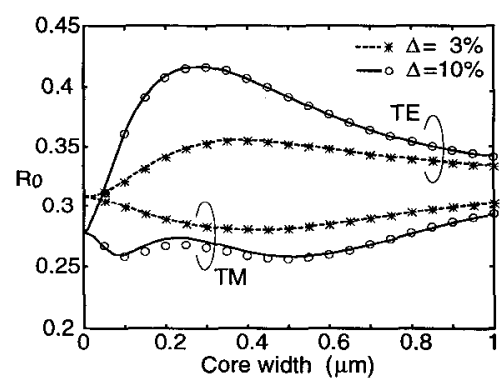

Fig. 4 Reflectivity of the fundamental mode as a function of the core width. Both TE and TM waves are shown. The index differences between the core and cladding are $3 \%$ and $10 \%$. Solid and dashed lines are calculated by the $P(3,3)$ approximant. Circles and stars are the results given in [6]. 